\title{
Short Sleep Duration by Occupation Group — 29 States, 2013-2014
}

\author{
Taylor M. Shockey, $\mathrm{MPH}^{1}$; Anne G. Wheaton, $\mathrm{PhD}^{2}$
}

The American Academy of Sleep Medicine and the Sleep Research Society have determined that adults require $\geq 7$ hours of sleep per day to promote optimal health (1). Short sleep duration ( $<7$ hours per day) has been linked to adverse health outcomes including cardiovascular disease, obesity, diabetes, depression, and anxiety, as well as safety issues related to drowsy driving and injuries $(1,2)$. Additional research has found that sleep duration varies by characteristics such as race, education, marital status, obesity, and cigarette smoking (3). Work-related factors such as job stress, work hours, shift work, and physically demanding work have been found to be associated with sleep duration and quality (4-6). All of these work factors vary by industry and occupation of employment, and the prevalence of short sleep duration has been shown to vary by broad industry and occupation category (7). To provide updated and more detailed information about which occupation groups have the highest prevalences of short sleep duration, CDC analyzed data from currently employed adults surveyed for the 2013 and 2014 Behavioral Risk Factor Surveillance System (BRFSS) in 29 states. Among 22 major occupation groups, the highest prevalences of short sleep duration were among workers in the following five groups: Production (42.9\%), Healthcare Support (40.1\%), Healthcare Practitioners and Technical (40.0\%), Food Preparation and Serving-Related (39.8\%), and Protective Service (39.2\%). The significant differences among occupation groups in the prevalence of short sleep duration suggest that work-related factors should be further evaluated as they might relate to sleep.

BRFSS is an annual, random-digit-dialed telephone survey of noninstitutionalized, U.S. civilian residents aged $\geq 18$ years. It is conducted by U.S. states and territories to gather data on health-related risk behaviors, chronic illnesses and conditions, and use of health-related services.* The BRFSS questionnaire is composed of a set of core questions that are asked by all states; in addition, states may choose from optional modules on specific subjects or include state-added questions. Twentynine states ${ }^{\dagger}$ administered the optional industry and occupation

\footnotetext{
${ }^{*}$ https://www.cdc.gov/brfss/annual_data/2013/pdf/overview_2013.pdf and https://www.cdc.gov/brfss/annual_data/2014/pdf/overview_2014.pdf.

$\dagger$ States providing data in 2013 and 2014: Illinois, Louisiana, Maryland, Massachusetts, Michigan, Minnesota, Mississippi, Montana, Nebraska, New Hampshire, New Jersey, New Mexico, New York, North Dakota, Oregon, Utah, and Washington. States contributing data only in 2013: California, Florida, Wisconsin, and Wyoming. States contributing data only in 2014: Colorado, Connecticut, Georgia, Idaho, Iowa, North Carolina, Tennessee, and Vermont. Washington and Wyoming's 2013 industry and occupation data are from stateadded questions, provided with permission of the two states' BRFSS coordinators.
}

module in 2013 or 2014. Response rates for BRFSS are calculated based on American Association of Public Opinion Research guidelines. The median response rate for all states, territories, and the District of Columbia was $46.4 \%$ in 2013 and $47.0 \%$ in 2014 , whereas the response rates for states included in analyses ranged from $31.1 \%$ to $59.2 \%$ in 2013 and from $33.0 \%$ to $57.6 \%$ in $2014 . \$$

To determine occupation, BRFSS participants who were employed for wages, self-employed, or out of work for $<1$ year were asked, "What kind of work do you do?" Participants' responses were recorded as free text and later coded to one of the 574 U.S. Bureau of Census (2002) occupation numeric codes by an auto-coding system or computer-assisted human coders. Because of the difficulty in reporting results for such a large number of occupations, and to protect participants' privacy, the 574 Bureau of Census codes were grouped into 93 two-digit detailed occupation groups used by CDC's National Center for Health Statistics to code occupations for the National Health Interview Survey, ${ }^{* *}$ and these detailed groups were collapsed into the 22 two-digit Standard Occupational Classification System major occupation groups created by the Bureau of Labor Statistics. ${ }^{\dagger \dagger}$ Respondents also were asked, "On average, how many hours of sleep do you get in a 24-hour period?" Responses to this question were dichotomized into $\geq 7$ hours of sleep (sufficient sleep) and $<7$ hours of sleep (short sleep duration).

Among the 412,829 BRFSS participants in 2013 and 2014, a total of 207,143 (50.2\%) were currently employed for wages, self-employed, or out of work for $<1$ year and were considered for analyses. After excluding 523 respondents $(0.3 \%)$ who were on active military duty, $249(0.1 \%)$ who were unpaid or retired workers, and 26,750 (12.9\%) with insufficient or missing information necessary for occupational coding, the final sample for analyses totaled 179,621 (86.7\% of the currently employed respondents). Prevalence of short sleep duration was calculated by the 22 major and 93 detailed occupation groups and adjusted for the following characteristics: age group (18-34 years, 35-44 years, 45-54 years, 55-64 years, and $\geq 65$ years); sex (male or female); race/ethnicity (non-Hispanic white, non-Hispanic black, Hispanic, and non-Hispanic

\footnotetext{
$\$$ https://www.cdc.gov/brfss/annual_data/2013/pdf/2013_dqr.pdf and https:// www.cdc.gov/brfss/annual_data/2014/pdf/2014_dqr.pdf.

I https://www.census.gov/people/io/methodology/.

** ftp://ftp.cdc.gov/pub/Health_Statistics/NCHS/Dataset_Documentation/ NHIS/2007/srvydesc.pdf.

$t^{\dagger}$ https://www.bls.gov/soc/major_groups.htm.
} 
other race or multiracial); marital status (married/member of an unmarried couple or divorced/widowed/separated/never married); and education level (less than high school diploma, graduated high school, some college, or college graduate). The adjusted prevalence estimates were obtained using logistic regression. F tests were used as a measure of association to determine statistical significance of the variables. All analyses were weighted to account for the survey design unless otherwise noted.

Overall, $36.5 \%$ of currently employed adults reported short sleep duration. The prevalence of short sleep duration among persons in the three youngest age groups was similar (18-34 years [37.7\%], 35-44 years [37.6\%], and 45-54 years $[37.4 \%])$ and lower among persons in the two oldest age groups (45-64 years [33.8\%] and $\geq 65$ years [29.2\%]). Among persons categorized by other characteristics, the highest prevalences were reported by men (37.5\%), non-Hispanic blacks (48.5\%), persons with some college education (40.0\%), and persons who were divorced, widowed, separated or never married (39.5\%) (Table 1).

Among the 22 major occupation groups, the highest prevalences of short sleep duration were among workers in the following five groups: Production (42.9\%), Healthcare Support (40.1\%), Healthcare Practitioners and Technical (40.0\%), Food Preparation and Serving-Related (39.8\%), and Protective Service (39.2\%). The two major occupation groups with the lowest prevalence of short sleep duration were Education, Training, and Library and Farming, Fishing, and Forestry (both 31.3\%) (Table 2).

Within the Protective Service major occupation group, the highest prevalence of short sleep duration was reported in the detailed group of firefighting and prevention workers (45.8\%). Within the Healthcare Support major group, the highest prevalences were reported in the detailed group of nursing, psychiatric, and home health aides (43.3\%). Among all major occupation groups, the detailed groups with the highest prevalences of short sleep duration were communications equipment operators $(58.2 \%)$, other transportation workers (54.0\%), and rail transportation workers (52.7\%). The detailed groups with the lowest prevalences of short sleep duration were air transportation workers (21.4\%) and religious workers (22.4\%) (Table 2).

For the 29 states, the weighted percentage of currently employed adults in any of the five major occupation groups with the highest prevalence of short sleep duration also was calculated. Among the states, the percentage of currently employed adults working in any of the five major occupation groups with the highest prevalence of short sleep duration ranged from $17.6 \%$ (Wyoming) to $26.8 \%$ (Mississippi) (Table 3).

\section{Discussion}

This study is the first to evaluate short sleep duration by 93 detailed occupation groups and at a multistate level. A previous study using National Health Interview Survey data found that within certain industries, production and transportation and material moving occupations had among the highest prevalences of short sleep duration, a finding that is consistent with the results of this study ( 7 ). Previous studies have shown that shift workers are more likely to experience disturbed sleep and excessive sleepiness, and to report a significantly higher prevalence of short sleep duration compared with day workers $(6,8)$. Shift work negatively influences health, by affecting the natural circadian rhythm, leading to irregularities in the sleep-wake cycle (8). The five major occupation groups with the highest prevalence of short sleep duration (Production, Healthcare Support, Healthcare Practitioners and Technical, Food Preparation and Serving-Related, and Protective Service) also have some of the highest prevalence rates of alternative shift work, ranging from $>35 \%$ of Healthcare Practitioners and Technical workers to $>50 \%$ of Food Preparation and ServingRelated workers (9).

Respondents working in detailed occupation groups within the major occupation group of Transportation and Material Moving reported a wide range in prevalences of short sleep duration, from air transportation workers $(21 \%)$ to other transportation workers (54\%). In 2011, the Federal Aviation Administration overhauled commercial airline pilot scheduling to ensure that pilots are rested before flying; this might account for the low prevalence of short sleep duration among air transportation workers. ${ }^{\$ S}$ In contrast, $53 \%$ of rail transportation workers reported short sleep duration. Although the Railroad Safety Improvement Act of 2008 mandated changes to the limitations on the number of hours railroad employees work, compliance with the bill is not required until 2018.99 Shift work and existing occupational regulations likely are important factors to consider regarding the results of this study.

The findings in this report are subject to at least four limitations. First, because BRFSS data are cross-sectional, it is not possible to determine temporal relationships. Second, BRFSS data are self-reported, and are therefore subject to recall and social desirability biases. Among certain occupations where sleep duration has been an issue and hours might be specified by regulation (e.g., transportation), there might be a greater sensitivity to this question and a bias toward reporting sufficient sleep. Third, because the data came from 29 states, the

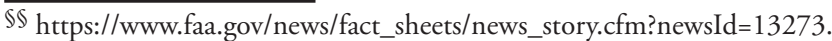
99 https://www.fra.dot.gov/eLib/details/L04320.
} 
TABLE 1. Prevalence of short sleep duration ( $<7$ hours of sleep per day) among currently employed adults, by selected characteristics Behavioral Risk Factor Surveillance System, 29 states, 2013-2014

\begin{tabular}{|c|c|c|c|}
\hline Characteristic & Unweighted no. & Weighted $\%$ of total sample population $(95 \% \mathrm{Cl})$ & Prevalence of short sleep $\%(95 \% \mathrm{Cl})$ \\
\hline \multicolumn{4}{|l|}{ Age group (yrs) } \\
\hline $18-34$ & 41,326 & $32.8(32.3-33.4)$ & $37.7(36.6-38.7)$ \\
\hline $35-44$ & 38,258 & $22.2(21.8-22.7)$ & $37.6(36.4-38.8)$ \\
\hline $45-54$ & 52,189 & $23.4(23.0-23.9)$ & $37.4(36.4-38.5)$ \\
\hline $55-64$ & 54,089 & $16.5(16.2-16.9)$ & $33.8(32.7-34.9)$ \\
\hline$\geq 65$ & 21,281 & $5.0(4.8-5.2)$ & $29.2(27.3-31.2)$ \\
\hline \multicolumn{4}{|l|}{ Sex } \\
\hline Men & 98,868 & $54.8(54.3-55.3)$ & $37.5(36.7-38.2)$ \\
\hline Women & 108,275 & $45.2(44.7-45.7)$ & $35.4(34.6-36.1)$ \\
\hline \multicolumn{4}{|l|}{ Race/Ethnicity } \\
\hline White, non-Hispanic & 165,130 & $63.4(62.8-63.9)$ & $33.5(32.9-34.0)$ \\
\hline Black, non-Hispanic & 13,523 & $11.1(10.7-11.5)$ & $48.5(46.8-50.2)$ \\
\hline Hispanic & 14,656 & $16.8(16.4-17.3)$ & $37.8(36.1-39.5)$ \\
\hline Other race or multiracial, non-Hispanic & 10,633 & $8.7(8.3-9.1)$ & $39.9(37.2-42.5)$ \\
\hline \multicolumn{4}{|l|}{ Education } \\
\hline Less than high school diploma & 8,863 & $10.7(10.3-11.2)$ & $37.4(35.3-39.5)$ \\
\hline Graduated high school & 48,818 & $24.9(24.5-25.4)$ & $38.9(37.8-39.9)$ \\
\hline Some college & 57,291 & $31.0(30.5-31.5)$ & $40.0(39.0-41.1)$ \\
\hline College graduate & 91,704 & $33.3(32.8-33.8)$ & $31.3(30.6-32.1)$ \\
\hline \multicolumn{4}{|l|}{ Marital status } \\
\hline Married/Member of an unmarried couple & 130,990 & $60.8(60.3-61.4)$ & $34.7(34.0-35.3)$ \\
\hline Divorced/Widowed/Separated/Never married & 74,856 & $39.2(38.6-39.7)$ & $39.5(38.6-40.4)$ \\
\hline \multicolumn{4}{|l|}{ State of residence } \\
\hline California & 3,706 & $18.0(17.6-18.4)$ & $37.3(35.3-39.4)$ \\
\hline Colorado & 4,590 & $2.8(2.8-2.9)$ & $29.2(27.4-31.0)$ \\
\hline Connecticut & 4,257 & $1.9(1.8-1.9)$ & $36.3(34.4-38.3)$ \\
\hline Florida & 12,982 & $9.1(8.9-9.3)$ & $38.9(37.2-38.3)$ \\
\hline Georgia & 2,857 & $4.8(4.7-4.9)$ & $38.8(36.5-41.1)$ \\
\hline Idaho & 2,628 & $0.8(0.7-0.8)$ & $32.2(29.7-34.8)$ \\
\hline Illinois & 5,403 & $6.5(6.4-6.6)$ & $35.9(34.2-37.6)$ \\
\hline lowa & 4,370 & $1.7(1.6-1.7)$ & $32.4(30.6-34.1)$ \\
\hline Louisiana & 5,449 & $2.1(2.1-2.2)$ & $37.4(35.5-39.2)$ \\
\hline Maryland & 13,210 & $3.2(3.2-3.3)$ & $40.6(39.2-42.0)$ \\
\hline Massachusetts & 15,405 & $3.5(3.5-3.6)$ & $35.2(34.1-36.4)$ \\
\hline Michigan & 9,811 & $4.6(4.5-4.7)$ & $40.3(39.1-41.6)$ \\
\hline Minnesota & 18,291 & $3.1(3.0-3.1)$ & $31.6(30.5-32.6)$ \\
\hline Mississippi & 4,735 & $1.3(1.3-1.4)$ & $35.9(34.0-37.9)$ \\
\hline Montana & 8,729 & $0.5(0.5-0.5)$ & $31.1(29.8-32.5)$ \\
\hline Nebraska & 11,011 & $1.0(1.0-1.0)$ & $32.5(31.1-34.0)$ \\
\hline New Hampshire & 6,628 & $0.7(0.7-0.7)$ & $32.5(31.0-34.1)$ \\
\hline New Jersey & 4,805 & $4.7(4.5-4.8)$ & $39.6(37.5-41.8)$ \\
\hline New Mexico & 8,571 & $0.9(0.9-1.0)$ & $33.1(31.7-34.6)$ \\
\hline New York & 3,826 & $9.8(9.6-10.1)$ & $40.1(38.0-42.3)$ \\
\hline North Carolina & 3,408 & $4.8(4.7-4.9)$ & $32.7(30.8-34.5)$ \\
\hline North Dakota & 9,082 & $0.4(0.4-0.4)$ & $32.0(30.6-33.5)$ \\
\hline Oregon & 4,966 & $1.8(1.8-1.9)$ & $31.8(30.1-33.5)$ \\
\hline Tennessee & 2,073 & $3.1(3.0-3.2)$ & $36.4(33.5-39.3)$ \\
\hline Utah & 15,806 & $1.5(1.4-1.5)$ & $33.9(32.9-34.8)$ \\
\hline Vermont & 3,945 & $0.4(0.4-0.4)$ & $29.7(28.0-31.4)$ \\
\hline Washington & 10,111 & $3.5(3.4-3.6)$ & $33.6(32.4-34.9)$ \\
\hline Wisconsin & 3,410 & $2.9(2.9-3.0)$ & $32.9(30.5-35.3)$ \\
\hline Wyoming & 3,078 & $0.3(0.3-0.3)$ & $29.2(27.1-31.4)$ \\
\hline Overall & 207,143 & $56.3(55.9-56.7)$ & $36.5(36.0-37.1)$ \\
\hline
\end{tabular}

Abbreviation: $\mathrm{Cl}=$ confidence interval.

results might not be representative of the national currently employed population. Finally, misclassification of occupation by respondents, interviewers or coders, although likely rare, is possible.
Short sleep duration among the U.S. working population has been estimated to result in a $\$ 411$ billion dollar annual cost to the economy, equivalent to $2.28 \%$ of the country's gross domestic product (10). In addition, among employed persons, 1.2 million working days are lost in the United 
TABLE 2. Prevalence of short sleep duration (<7 hours of sleep per day) among currently employed adults, by Standard Occupational Classification (SOC) System major occupation groups and detailed occupation groups* - Behavioral Risk Factor Surveillance System (BRFSS), 29 states, 2013-2014

\begin{tabular}{|c|c|c|c|c|}
\hline Major occupation group (SOC code)/Detailed occupation group & Unweighted no. & Unadjusted \% (95\% Cl) & Adjusted ${ }^{\dagger} \%(95 \% \mathrm{Cl})$ & CV for adjusted $\%$ \\
\hline Production (51) & 7,605 & $44.6(42.0-47.2)$ & $42.9(40.3-45.4)$ & 0.03 \\
\hline Printing workers & 216 & $52.3(38.1-66.6)$ & $50.9(37.1-64.6)$ & 0.14 \\
\hline Plant and system operators & 503 & $52.3(40.4-64.3)$ & $49.6(38.7-60.5)$ & 0.11 \\
\hline Supervisors, production workers & 546 & $50.3(39.9-60.8)$ & $48.9(39.0-58.9)$ & 0.10 \\
\hline Other production occupations & 2,671 & $47.1(42.9-51.3)$ & $45.6(41.5-49.8)$ & 0.05 \\
\hline Metal workers and plastic workers & 1,478 & $45.3(40.5-50.2)$ & $44.0(39.2-49.0)$ & 0.06 \\
\hline Woodworkers & 199 & $40.3(24.2-56.4)$ & $39.2(25.9-54.4)$ & 0.19 \\
\hline Assemblers and fabricators & 811 & $39.4(32.2-46.6)$ & $36.8(29.9-44.2)$ & 0.10 \\
\hline Food processing workers & 543 & $37.9(28.7-47.1)$ & $35.9(27.5-45.3)$ & 0.13 \\
\hline Textile, apparel, and furnishings workers & 638 & $34.6(24.7-44.6)$ & $34.2(24.9-44.8)$ & 0.15 \\
\hline Healthcare Support (31) & 4,328 & $42.7(39.4-46.1)$ & $40.1(36.7-43.5)$ & 0.04 \\
\hline Nursing, psychiatric, and home health aides & 2,484 & $47.8(43.3-52.3)$ & $43.3(38.9-47.8)$ & 0.05 \\
\hline Other healthcare support occupations & 1,732 & $35.8(30.6-41.0)$ & $35.7(30.5-41.3)$ & 0.08 \\
\hline Occupational and physical therapist assistants and aides & 112 & $30.5(15.8-45.1)$ & $32.8(19.7-49.4)$ & 0.24 \\
\hline Healthcare Practitioners and Technical (29) & 14,975 & $38.1(35.9-40.2)$ & $40.0(37.8-42.2)$ & 0.03 \\
\hline Health technologists and technicians & 3,218 & $41.0(37.2-44.9)$ & $40.4(36.7-44.3)$ & 0.05 \\
\hline Health diagnosing and treating practitioners & 11,589 & $37.2(34.7-39.7)$ & $39.7(37.0-42.4)$ & 0.04 \\
\hline Other healthcare practitioners and technical occupations & 168 & $33.4(15.6-51.2)$ & $35.1(21.0-52.6)$ & 0.24 \\
\hline Food Preparation and Serving-Related (35) & 5,413 & $42.4(39.1-45.6)$ & $39.8(36.6-43.0)$ & 0.04 \\
\hline Supervisors, food preparation, and serving workers & 910 & $53.1(44.8-61.4)$ & $48.9(40.6-57.3)$ & 0.09 \\
\hline Cooks and food preparation workers & 2,162 & $44.3(38.9-49.7)$ & $41.4(36.3-46.8)$ & 0.07 \\
\hline Food and beverage serving workers & 1,876 & $37.4(32.4-42.3)$ & $36.1(31.4-41.1)$ & 0.07 \\
\hline Other food preparation and serving related workers & 465 & $33.0(23.0-43.1)$ & $30.8(21.7-41.6)$ & 0.17 \\
\hline Protective Service (33) & 3,462 & $42.4(38.6-46.2)$ & $39.2(35.6-43.0)$ & 0.05 \\
\hline Firefighting and prevention workers & 534 & $48.0(39.0-56.9)$ & $45.8(37.1-54.7)$ & 0.10 \\
\hline Law enforcement officers & 1,591 & $42.2(36.8-47.7)$ & $39.8(34.6-45.3)$ & 0.07 \\
\hline Other protective service workers & 1,129 & $41.9(35.0-48.7)$ & $37.7(31.4-44.4)$ & 0.09 \\
\hline First-line supervisors/managers, protective service workers & 208 & $26.4(13.2-39.6)$ & $23.7(13.0-39.3)$ & 0.28 \\
\hline Transportation and Material Moving (53) & 8,014 & $42.3(39.7-44.9)$ & $39.1(36.6-41.7)$ & 0.03 \\
\hline Other transportation workers & 138 & $56.5(39.4-73.7)$ & $54.0(35.9-71.2)$ & 0.17 \\
\hline Rail transportation workers & 227 & $54.5(39.2-69.8)$ & $52.7(37.4-67.4)$ & 0.15 \\
\hline Supervisors, transportation and material moving employees & 141 & $48.0(29.1-66.9)$ & $43.3(26.0-62.4)$ & 0.22 \\
\hline Material moving workers & 2,337 & $44.2(39.5-48.8)$ & $40.5(36.0-45.1)$ & 0.06 \\
\hline Motor vehicle operators & 4,823 & $41.5(38.0-44.9)$ & $38.5(35.2-41.9)$ & 0.04 \\
\hline Water transportation workers & 99 & $30.0(14.8-45.2)$ & $31.5(18.4-48.4)$ & 0.25 \\
\hline Air transportation workers & 249 & $20.6(11.6-29.7)$ & $21.4(13.3-32.8)$ & 0.23 \\
\hline Personal Care and Service (39) & 5,907 & $38.9(35.3-42.5)$ & $37.5(34.0-41.1)$ & 0.05 \\
\hline Supervisors, personal care and service workers & 153 & $32.8(14.5-51.0)$ & $34.3(17.7-55.9)$ & 0.30 \\
\hline Animal care and service workers & 289 & $34.1(19.2-48.9)$ & $35.3(22.0-51.3)$ & 0.22 \\
\hline Entertainment attendants and related workers & 219 & $51.7(25.1-78.3)$ & $48.2(27.1-69.9)$ & 0.24 \\
\hline Personal appearance workers & 1,114 & $34.1(27.3-40.9)$ & $31.7(25.4-38.9)$ & 0.11 \\
\hline Transportation, tourism, and lodging attendants & 236 & $41.7(27.6-55.7)$ & $36.4(25.1-49.4)$ & 0.17 \\
\hline Other personal care and service workers & 3,876 & $39.4(35.3-43.5)$ & $38.5(34.4-42.7)$ & 0.05 \\
\hline Funeral service workers & 20 & $-\S$ & - & 0.18 \\
\hline Installation, Maintenance, and Repair (49) & 5,328 & $38.4(35.2-41.5)$ & $36.6(33.5-39.8)$ & 0.04 \\
\hline Other installation, maintenance, and repair occupations & 1,946 & $39.9(34.7-45.2)$ & $38.7(33.6-44.1)$ & 0.07 \\
\hline Electrical and electronic equipment mechanics, installers, and repairers & 713 & $39.1(30.4-47.9)$ & $36.6(28.4-45.7)$ & 0.12 \\
\hline Vehicle and mobile equipment mechanics, installers, and repairers & 2,431 & $37.7(33.1-42.4)$ & $36.0(31.6-40.7)$ & 0.06 \\
\hline Supervisors of installation, maintenance, and repair workers & 238 & $27.2(16.1-38.3)$ & $27.5(18.0-39.6)$ & 0.20 \\
\hline Office and Administrative Support (43) & 21,406 & $36.6(34.9-38.3)$ & $36.5(34.8-38.3)$ & 0.02 \\
\hline Communications equipment operators & 109 & $59.0(43.1-74.9)$ & $58.2(42.6-72.3)$ & 0.13 \\
\hline Material recording, scheduling, dispatching, and distribution workers & 2,584 & $46.2(41.1-51.3)$ & $44.6(39.5-49.9)$ & 0.06 \\
\hline Other office and administrative support workers & 5,325 & $35.7(32.0-39.3)$ & $36.0(32.3-39.7)$ & 0.05 \\
\hline Information and record clerks & 4,279 & $36.9(33.5-40.3)$ & $35.9(32.5-39.3)$ & 0.05 \\
\hline Financial clerks & 3,539 & $34.8(30.3-39.3)$ & $35.3(30.9-40.0)$ & 0.07 \\
\hline Supervisors, office and administrative support workers & 2,139 & $31.6(27.4-35.9)$ & $33.3(29.1-37.8)$ & 0.07 \\
\hline Secretaries and administrative assistants & 3,431 & $31.7(28.1-35.3)$ & $32.4(29.0-36.1)$ & 0.06 \\
\hline Business and Financial Operations (13) & 7,811 & $33.9(31.1-36.8)$ & $36.1(33.3-39.0)$ & 0.04 \\
\hline Business operations specialists & 3,734 & $34.7(30.5-39.0)$ & $36.0(32.2-40.0)$ & 0.06 \\
\hline Financial specialists & 4,077 & $33.1(29.2-36.9)$ & $36.0(32.2-40.0)$ & 0.06 \\
\hline Building and Grounds Cleaning and Maintenance (37) & 6,265 & $38.0(35.1-40.9)$ & $36.0(33.2-39.0)$ & 0.04 \\
\hline Supervisors, building and grounds cleaning and maintenance workers & 415 & $42.4(33.7-51.2)$ & $41.2(33.3-49.6)$ & 0.10 \\
\hline Building cleaning and pest control workers & 4,750 & $40.2(36.8-43.6)$ & $38.2(34.8-41.6)$ & 0.04 \\
\hline Grounds maintenance workers & 1,100 & $30.4(24.1-36.6)$ & $28.8(23.1-35.3)$ & 0.11 \\
\hline
\end{tabular}

See table footnotes on next page. 
TABLE 2. (Continued) Prevalence of short sleep duration ( $<7$ hours of sleep per day) among currently employed adults, by Standard Occupational Classification (SOC) System major occupation groups and detailed occupation groups* — Behavioral Risk Factor Surveillance System (BRFSS), 29 states, 2013-2014

\begin{tabular}{|c|c|c|c|c|}
\hline Major occupation group (SOC code)/Detailed occupation group & Unweighted no. & Unadjusted \% (95\% Cl) & Adjusted ${ }^{\dagger} \%(95 \% \mathrm{Cl})$ & CV for adjusted \% \\
\hline Arts, Design, Entertainment, Sports, and Media (27) & 4,124 & $33.5(28.7-38.3)$ & $35.5(31.1-40.2)$ & 0.06 \\
\hline Art and design workers & 1,569 & $38.0(28.6-47.4)$ & $39.0(31.0-47.7)$ & 0.11 \\
\hline Entertainers and performers, sports and related workers & 769 & $33.7(23.8-43.6)$ & $34.8(26.1-44.6)$ & 0.14 \\
\hline Media and communication workers & 1,392 & $29.3(23.7-34.8)$ & $33.6(28.1-39.6)$ & 0.09 \\
\hline Media and communication equipment workers & 394 & $28.4(17.6-39.2)$ & $29.3(19.6-41.3)$ & 0.19 \\
\hline Management (11) & 21,808 & $33.8(32.1-35.4)$ & $35.4(33.7-37.2)$ & 0.03 \\
\hline Chief, executives; general and operations managers; legislators & 2,529 & $33.4(29.4-37.4)$ & $36.3(32.2-40.6)$ & 0.06 \\
\hline Operations specialties managers & 3,167 & $34.1(30.3-37.8)$ & $35.6(31.8-39.6)$ & 0.06 \\
\hline Other management occupations & 14,795 & $34.0(31.9-36.2)$ & $35.3(33.0-37.5)$ & 0.03 \\
\hline Advertising, marketing, promotions, public relations, and sales managers & 1,317 & $31.7(25.8-37.5)$ & $34.1(28.4-40.3)$ & 0.09 \\
\hline Legal (23) & 2,694 & $31.1(27.3-34.8)$ & $34.5(30.6-38.5)$ & 0.06 \\
\hline Legal support workers & 758 & $35.9(28.5-43.2)$ & $37.5(30.4-45.1)$ & 0.10 \\
\hline Lawyers, judges, and related workers & 1,936 & $29.1(24.7-33.5)$ & $32.9(28.3-37.7)$ & 0.07 \\
\hline Construction and Extraction (47) & 9,208 & $36.1(33.8-38.3)$ & $34.5(32.2-36.9)$ & 0.03 \\
\hline Extraction workers & 575 & $46.0(36.7-55.3)$ & $45.3(36.3-54.7)$ & 0.10 \\
\hline Construction trades workers & 6,975 & $36.2(33.6-38.7)$ & $34.6(32.0-37.3)$ & 0.04 \\
\hline Other construction and related workers & 458 & $34.3(23.6-45.0)$ & $34.5(24.4-46.2)$ & 0.16 \\
\hline Supervisors, construction and extraction workers & 1,184 & $34.4(28.8-40.1)$ & $34.2(28.8-40.1)$ & 0.08 \\
\hline Helpers, constructions trades & 16 & - & - & 0.89 \\
\hline Sales and Related (41) & 16,526 & $34.9(33.0-36.7)$ & $34.4(32.6-36.3)$ & 0.03 \\
\hline Supervisors, sales workers & 3,332 & $36.0(31.8-40.2)$ & $36.0(32.0-40.2)$ & 0.06 \\
\hline Sales representatives, services & 2,214 & $33.7(28.9-38.5)$ & $35.4(30.7-40.4)$ & 0.07 \\
\hline Retail sales workers & 7,243 & $36.3(33.4-39.1)$ & $34.4(31.7-37.3)$ & 0.04 \\
\hline Other sales and related workers & 2,408 & $32.4(27.3-37.4)$ & $33.5(28.6-38.8)$ & 0.08 \\
\hline Sales representatives, wholesale and manufacturing & 1,329 & $29.1(23.8-34.5)$ & $30.3(25.1-36.1)$ & 0.09 \\
\hline Architecture and Engineering (17) & 4,886 & $32.6(29.3-35.8)$ & $34.3(31.0-37.9)$ & 0.05 \\
\hline Drafters, engineering, and mapping technicians & 847 & $42.0(34.1-49.9)$ & $40.5(33.0-48.5)$ & 0.10 \\
\hline Architects, surveyors, and cartographers & 432 & $33.8(21.6-46.0)$ & $36.2(24.6-49.7)$ & 0.18 \\
\hline Engineers & 3,607 & $29.8(26.2-33.5)$ & $32.2(28.4-36.3)$ & 0.06 \\
\hline Computer and Mathematical (15) & 5,591 & $33.3(30.5-36.1)$ & $33.8(31.1-36.7)$ & 0.04 \\
\hline Mathematical science occupations & 278 & $36.8(25.6-48.0)$ & $38.1(27.4-50.2)$ & 0.15 \\
\hline Computer specialists & 5,313 & $33.2(30.3-36.1)$ & $33.6(30.7-36.6)$ & 0.04 \\
\hline Life, Physical, and Social Science (19) & 3,265 & $30.2(26.5-34.0)$ & $33.6(29.7-37.7)$ & 0.06 \\
\hline Life, physical, and social science technicians & 552 & $41.3(32.0-50.6)$ & $41.8(32.9-51.2)$ & 0.11 \\
\hline Physical scientists & 929 & $28.8(22.6-35.0)$ & $32.4(25.9-39.6)$ & 0.11 \\
\hline Social scientists and related workers & 970 & $27.9(20.3-35.4)$ & $32.3(24.8-40.9)$ & 0.13 \\
\hline Life scientists & 814 & $23.8(17.9-29.8)$ & $26.8(20.9-33.6)$ & 0.12 \\
\hline Community and Social Services (21) & 4,224 & $31.3(26.9-35.7)$ & $32.2(27.7-37.1)$ & 0.07 \\
\hline Counselors, social workers, and other community and social service specialists & 3,322 & $33.6(28.5-38.7)$ & $34.0(28.8-39.7)$ & 0.08 \\
\hline Religious workers & 902 & $20.5(15.2-25.7)$ & $22.4(17.2-28.6)$ & 0.13 \\
\hline Education, Training, and Library (25) & 15,249 & $27.9(26.2-29.7)$ & $31.3(29.2-33.4)$ & 0.04 \\
\hline Postsecondary teachers & 2,351 & $21.8(18.4-25.3)$ & $25.4(21.7-29.4)$ & 0.08 \\
\hline Primary, secondary, and special education school workers & 9,806 & $29.0(26.7-31.2)$ & $32.5(29.9-35.1)$ & 0.04 \\
\hline Other teachers and instructors & 834 & $23.6(17.1-30.2)$ & $25.2(19.0-32.7)$ & 0.14 \\
\hline Librarians, curators, and archivists & 628 & $26.2(18.5-33.9)$ & $30.3(22.9-38.8)$ & 0.14 \\
\hline Other education, training, and library occupations & 1,630 & $31.6(25.8-37.4)$ & $32.5(26.8-38.8)$ & 0.10 \\
\hline Farming, Fishing, and Forestry (45) & 1,532 & $32.1(24.5-38.7)$ & $31.3(25.0-38.4)$ & 0.11 \\
\hline Fishing and hunting workers & 82 & $35.1(16.0-54.2)$ & $36.6(20.4-56.5)$ & 0.26 \\
\hline Agricultural workers & 1,210 & $31.0(23.6-38.4)$ & $30.2(23.1-38.3)$ & 0.13 \\
\hline Forest, conservations, and logging workers & 179 & - & - & 0.31 \\
\hline Supervisors, farming, fishing, and forestry workers & 61 & - & - & 0.41 \\
\hline All occupation groups & 179,621 & $36.5(35.9-37.1)$ & - & - \\
\hline
\end{tabular}

Abbreviations: $\mathrm{Cl}=$ confidence interval; $\mathrm{CV}=$ coefficient of variation.

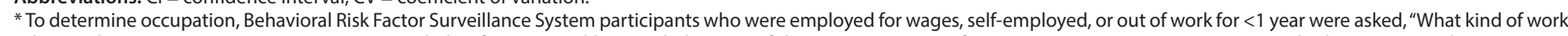

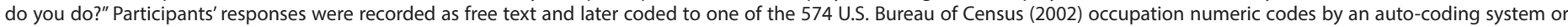

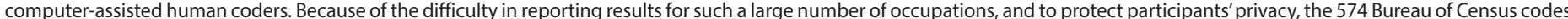

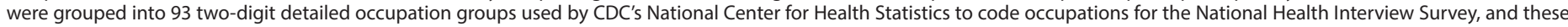

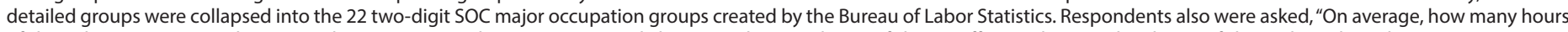
of sleep do you get in a 24-hour period?" Responses to this question were dichotomized into $\geq 7$ hours of sleep (sufficient sleep) and $<7$ hours of sleep (short sleep duration).

+ Adjusted by sex, race/ethnicity, marital status, age group, and education level.

$\S$ Estimates were suppressed because they did not meet the statistical reliability standards of BRFSS (i.e., cell size was $<50$ participants or CV $>0.30$ ). 
States each year because of sleep deprivation. It is estimated that if persons who sleep $<6$ hours per day began sleeping for $6-7$ hours per day, approximately $\$ 226$ billion could be added to the U.S. economy (10). A goal of Healthy People 2020 is to "increase public knowledge of how adequate sleep and treatment of sleep disorders improve health, productivity, wellness, quality of life, and safety on roads and in the workplace," with a specific objective to increase the proportion of adults getting sufficient sleep. ${ }^{* * *}$

CDC's National Institute for Occupational Safety and Health has developed educational resources on shift work and long working hours for managers, workers, and the public. ${ }^{\dagger \dagger}$ The materials include interactive training for nurses, emergency responders, and truck drivers, as well as information for aviation and railroad employees, methods for improving shiftwork schedules, and individual coping strategies. Time at work continues to increase, with the United States having the longest annual working hours among all

\footnotetext{
*** https://www.healthypeople.gov/2020/topics-objectives/topic/sleep-health. $t_{\dagger \dagger}$ https://www.cdc.gov/niosh/topics/workschedules/education.html.
}

wealthy industrialized countries (7). Job characteristics, such as schedules, stress, and physical output, should be evaluated in an effort to improve worker sleep duration and overall health.

\section{Acknowledgments}

Aaron Sussell, Sara Luckhaupt, Jan Birdsey, Jeff Purdin, Pam Schumacher, Susan Burton, Division of Surveillance, Hazard Evaluations, and Field Studies, National Institute for Occupational Safety and Health, CDC; 29 Behavioral Risk Factor Surveillance System state coordinators.

${ }^{1}$ Division of Surveillance, Hazard Evaluations, and Field Studies, National Institute for Occupational Safety and Health, CDC; ${ }^{2}$ Division of Population Health, National Center for Chronic Disease Prevention and Health Promotion, CDC.

Corresponding author: Taylor M. Shockey, tshockey@cdc.gov, 513-841-4239.

\section{References}

1. Watson NF, Badr MS, Belenky G, et al. Joint consensus statement of the American Academy of Sleep Medicine and Sleep Research Society on the recommended amount of sleep for a healthy adult: methodology and discussion. J Clin Sleep Med 2015;11:931-52.

TABLE 3. Weighted percentage of currently employed adults in the five major occupation groups of the Standard Occupational Classification System with the highest prevalence of short sleep duration ( $<7$ hours of sleep per day), by state - Behavioral Risk Factor Surveillance System, 29 states, 2013-2014

\begin{tabular}{|c|c|c|c|c|c|c|}
\hline \multirow[b]{2}{*}{ State } & \multicolumn{6}{|c|}{ Top five major occupation groups } \\
\hline & Production & $\begin{array}{l}\text { Healthcare } \\
\text { Support }\end{array}$ & $\begin{array}{c}\text { Healthcare } \\
\text { Practitioners and } \\
\text { Technical }\end{array}$ & $\begin{array}{c}\text { Food } \\
\text { Preparation and } \\
\text { Serving-Related }\end{array}$ & $\begin{array}{l}\text { Protective } \\
\text { Service }\end{array}$ & $\begin{array}{c}\text { Any of the top five } \\
\text { major occupation } \\
\text { groups }\end{array}$ \\
\hline Mississippi & 7.7 & 2.5 & 8.2 & 5.2 & 3.2 & 26.8 \\
\hline Tennessee & 8.0 & 2.6 & 6.6 & 5.6 & 2.5 & 25.3 \\
\hline Wisconsin & 10.1 & 3.1 & 6.2 & 3.9 & 2.0 & 25.3 \\
\hline Louisiana & 4.8 & 3.3 & 7.7 & 6.2 & 3.0 & 25.0 \\
\hline Michigan & 8.1 & 3.2 & 7.4 & 4.2 & 1.9 & 24.8 \\
\hline North Carolina & 5.7 & 3.1 & 7.2 & 4.2 & 2.5 & 22.7 \\
\hline lowa & 7.5 & 3.2 & 7.0 & 3.1 & 1.5 & 22.3 \\
\hline New York & 3.9 & 3.1 & 8.0 & 4.8 & 2.5 & 22.3 \\
\hline Illinois & 6.5 & 3.0 & 6.3 & 4.4 & 1.9 & 22.1 \\
\hline Minnesota & 6.1 & 2.8 & 8.1 & 3.9 & 1.2 & 22.1 \\
\hline Oregon & 5.8 & 2.5 & 6.8 & 4.1 & 2.5 & 21.7 \\
\hline Massachusetts & 3.7 & 2.8 & 9.6 & 3.6 & 1.8 & 21.5 \\
\hline Florida & 3.2 & 3.0 & 6.8 & 5.4 & 3.1 & 21.5 \\
\hline Vermont & 6.0 & 2.4 & 6.9 & 4.5 & 1.6 & 21.4 \\
\hline Connecticut & 4.7 & 3.0 & 8.0 & 3.4 & 2.3 & 21.4 \\
\hline Nebraska & 6.0 & 2.6 & 7.3 & 3.8 & 1.6 & 21.3 \\
\hline North Dakota & 5.0 & 2.8 & 6.8 & 4.3 & 1.9 & 20.8 \\
\hline Idaho & 5.9 & 2.7 & 5.1 & 4.4 & 2.4 & 20.5 \\
\hline New Hampshire & 4.7 & 3.1 & 7.6 & 3.1 & 1.7 & 20.2 \\
\hline Montana & 4.8 & 2.5 & 5.8 & 4.4 & 2.4 & 19.9 \\
\hline California & 5.1 & 1.7 & 5.4 & 5.0 & 2.1 & 19.3 \\
\hline New Jersey & 3.2 & 2.9 & 7.6 & 2.4 & 3.2 & 19.3 \\
\hline Washington & 4.6 & 2.6 & 6.0 & 4.2 & 1.8 & 19.2 \\
\hline New Mexico & 3.6 & 2.6 & 6.2 & 3.8 & 2.9 & 19.1 \\
\hline Georgia & 5.2 & 1.6 & 5.6 & 4.6 & 1.8 & 18.8 \\
\hline Maryland & 1.9 & 2.3 & 6.9 & 4.2 & 3.5 & 18.8 \\
\hline Utah & 5.2 & 2.4 & 5.6 & 3.3 & 2.2 & 18.7 \\
\hline Colorado & 3.6 & 2.2 & 6.0 & 4.2 & 2.5 & 18.5 \\
\hline Wyoming & 5.2 & 1.7 & 5.2 & 3.2 & 2.3 & 17.6 \\
\hline
\end{tabular}


2. Liu Y, Wheaton AG, Chapman DP, Croft JB. Sleep duration and chronic diseases among U.S. adults age 45 years and older: evidence from the 2010 Behavioral Risk Factor Surveillance System. Sleep 2013;36:1421-7.

3. Liu Y, Wheaton AG, Chapman DP, Cunningham TJ, Lu H, Croft JB. Prevalence of healthy sleep duration among adults_-United States, 2014. MMWR Morb Mortal Wkly Rep 2016;65:137-41. http://dx.doi. org/10.15585/mmwr.mm6506a1

4. Ota A, Masue T, Yasuda N, Tsutsumi A, Mino Y, Ohara H. Association between psychosocial job characteristics and insomnia: an investigation using two relevant job stress models-the demand-control-support (DCS) model and the effort-reward imbalance (ERI) model. Sleep Med 2005;6:353-8. http://dx.doi.org/10.1016/j.sleep.2004.12.008

5. Åkerstedt T, Fredlund P, Gillberg M, Jansson B. Work load and work hours in relation to disturbed sleep and fatigue in a large representative sample. J Psychosom Res 2002;53:585-8. http://dx.doi.org/10.1016/ S0022-3999(02)00447-6

6. Luckhaupt SE. Short sleep duration among workers-United States, 2010. MMWR Morb Mortal Wkly Rep 2012;61:281-5.

7. Luckhaupt SE, Tak S, Calvert GM. The prevalence of short sleep duration by industry and occupation in the National Health Interview Survey. Sleep 2010;33:149-59.

8. Drake CL, Roehrs T, Richardson G, Walsh JK, Roth T. Shift work sleep disorder: prevalence and consequences beyond that of symptomatic day workers. Sleep 2004;27:1453-62.

9. Alterman T, Luckhaupt SE, Dahlhamer JM, Ward BW, Calvert GM. Prevalence rates of work organization characteristics among workers in the U.S.: data from the 2010 National Health Interview Survey. Am J Ind Med 2013;56:647-59. http://dx.doi.org/10.1002/ajim.22108

10. Hafner M, Stepanek M, Taylor J, et al. Why sleep matters - the economic costs of insufficient sleep: a cross-country comparative analysis. Santa Monica, CA: RAND Corporation; 2016. http://www.rand.org/pubs/ research_reports/RR1791.html

\section{Summary \\ What is already known about this topic?}

Shift work and other work factors influence sleep duration and sleep quality, which have a direct effect on worker health and safety. Previous research has found that workers in production, health care, protective service, transportation and material moving, and food preparation and serving-related fields are more likely to be shift workers. In addition, production and transportation and material moving occupations have been associated with higher prevalences of short sleep duration.

What is added by this report?

Analysis of 2013 and 2014 Behavioral Risk Factor Surveillance System data conducted to examine 93 detailed occupation groups in 29 states found that the prevalence of $<7$ hours of sleep per day (short sleep duration) ranged from $21.4 \%$ among air transportation workers to $58.2 \%$ among communications equipment workers. The percentage of workers employed in at least one of the five occupations with the highest prevalence of short sleep duration ranged from $17.6 \%$ in Wyoming to $26.8 \%$ in Mississippi.

What are the implications for public health practice?

Significant differences were found in the prevalence of short sleep duration among occupation groups. Workers in occupations with high prevalences of short sleep duration might be most at risk for sleep-related accidents and adverse health outcomes associated with short sleep duration. Work-related factors should be further evaluated in the context of short sleep duration to guide prevention efforts. 